\title{
Conservation of heritage buildings in Mashhad: on the impact of climate change and the urban heat island effect
}

\author{
Hamed Hedayatnia ${ }^{1}$, Marijke Steeman ${ }^{1}$, Nathan Van Den Bossche ${ }^{1}$ \\ ${ }^{1}$ Building physics Group, Faculty of Engineering and Architecture, Ghent University, 9000-Gent, Belgium
}

\begin{abstract}
The first step to preserve the historical heritage against global warming effects is understanding how this phenomenon affects the process of material degradation. This impact can be intensified in dense metropolitan areas by the impact of the urban heat island. This research aims to understand whether the cultural heritage located in Mashhad suffers from this phenomenon or not. By comparing the modelled climate data with the observations recorded for Mashhad meteorological station and another station located in a rural area close to the city, the existence of the urban heat island and timing of the phenomenon intensification has been analysed. Our study results show that the rising air temperature combined with the significant city sprawling during 1980-1990 created the urban heat island that the heritage construction suffered.
\end{abstract}

.Peer-review under the responsibility of the organizing committee of the ICMB21.

Keywords: Climate change; Urban Heat Island; Timurid; Mashhad; Heritage; Hyghrothermal.

\begin{tabular}{|ll}
\hline Nomenclature & \\
UHI & Urban heat island \\
RH & Relative humidity \\
ALARO & Combination of ALADIN (Aire Limitée Adaptation Dynamique Développement International) and AROME \\
(Application de la Recherche à l'Opérationnel à Meso-Echelle)
\end{tabular}

\section{Introduction}

Given that Mashhad had been the Timurid Empire's capital city during the reign of king Shahrokh, the son of Timur, the northeastern part of Iran hosts some valuable Timurid heritage buildings. Acquiring knowledge on the building materials and hygrothermal behaviour of components subjected to climate change is the first step toward assessing adaptive approaches to preserve historical buildings. A reliable numerical prediction of material moisture response essentially needs proper knowledge of climatic parameters like urban heat island (UHI) effects.

Mashhad is the second biggest city in Iran, a dense metropolitan area sprawling fast, particularly during 1980-1990 (Fig-1). Given the existence of the well-known urban heat island phenomenon (UHI), this intense urbanization might contribute to additional warming at this location. As the land use is kept constant during the climate simulation runs, the local effect of urbanization is not present in the modelled climate, which might explain the slower heating rate of the climate model in Mashhad compared with the rural station.

The urban heat island effect is most apparent at night when buildings release the solar-induced heat absorbed during the day. At the time of the maximum temperature, the urban effect causes a slight cooling, resulted by shading and aerosols to thermal inertia differences between city and country. Agricultural development can affect the climate by increasing evaporation during the day (decreasing the maximum temperature) and would also cause an increase in minimum temperature: irrigation increases the soil's heat capacity. Thus, both urbanisation and agriculture impacts could be consistent with a significant increase in the minimum temperature and slight decrease or a smooth rise in maximum temperature and reduce the diurnal temperature range (DTR) [1]. These effects are most anticipated for the observations so, the ALARO-0 model output, which formerly had been well evaluated over the region [2] has been selected to compare with the observation to assess the impacts of urban heat island on climatic 


\section{ICMB21}

parameters. In this analysis, the RCM data originates from the ALARO-0 runs at a spatial resolution of $25 \mathrm{~km}$ across the central Asia domain available at a time-frequency of 1-hour.

Moreover, data recorded in a rural station near Mashhad(Golmakan) has been compared with the data collected from Mashhad station to show the impact of urbanization on climate.

\section{Results and conclusion}

Daily DTR values have been calculated by subtracting daily minimum temperature from the daily maximum temperature to compare the results with the city sprawling rate to show the notable impact of UHI on the surface temperature during the 1980s (fig1). As it can be observed, Mashhad experienced an almost explosive sprawl in population and accordingly in urbanisation during the 1980s. Comparison between the modelled DTR, minimum and maximum values with the observations during the studied period shows that during the 1980s, there is a significant decrease in DTR trend for the observations whereas, during the following decades, the observed DTR values are significantly consistent with the modelled DTR computed data. Analysing the min-max trends can aim to confirm the impact of UHI. Correlated with the mentioned theory, a sharp increase in daily minimum temperature for the observed data, considerably sharper than the models, during the 1980s followed by a growing trend in the next decade, correlated with the modelled data, and a smooth increase in maximum temperature for the observations that are consistent with both models dataset in result, the urban heat island primarily affect the climate over the city during 1980's decade.

In the next step, simulations have been performed with three different climate conditions based on the observations recorded in urban and rural stations, respectively and the ALARO-0 model (representing the climate without the UHI effects). Moreover, due to the vast increase in wind velocity (despite the decremental trend of the precipitation), the wind-driven rain has increased and caused a rise in the moisture content of the external walls accordingly, it clearly shows a significant increase in damage risks that is continued to the future since no mitigating actions have yet been considered.
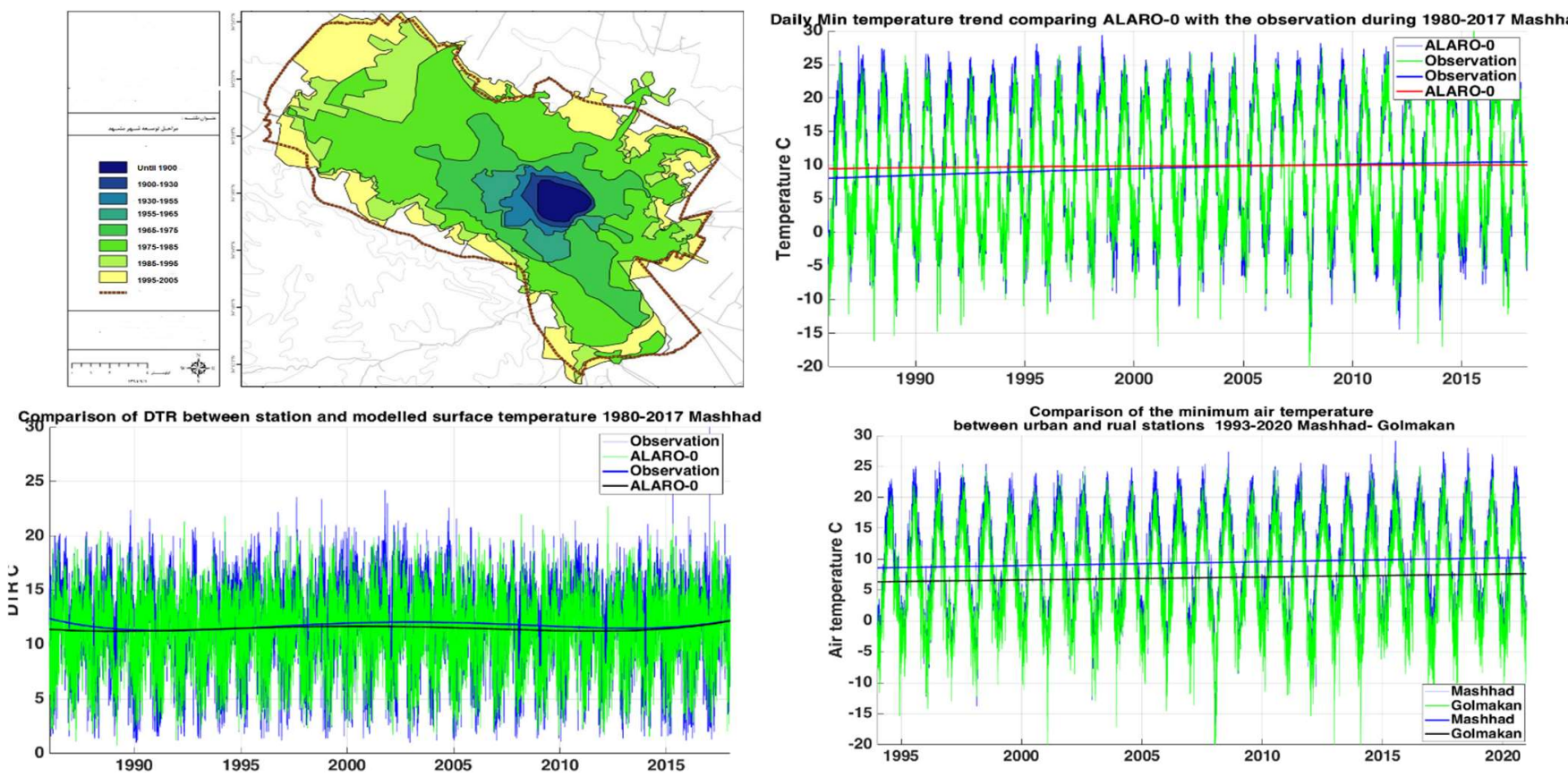

Figure1. Mashhad sprawling map(top-left), Minimum temperature comparison with the model(top-right), DTR comparison with the model(bot-left) and minimum temperature compared with the rural station.

\section{References}

[1] Kalnay, E., \& Kay, M. (2003). Impact of urbanization and land-use change on climate. Nature 423,528-531.

[2] Hedayatnia, H., \& Van Den Bossche, N., \& Steeman, M. (2020). The Impact of Climate Change on Material Degradation Criteria in Heritage over Iran: Regional Climate Model Evaluation. E3S Web of Conferences 172(3):02006. 